\title{
ГЕМОДИНАМИКА МАТКИ И СОСТОЯНИЕ ОВАРИАЛЬНОГО РЕЗЕРВА В ОЦЕНКЕ ЭФФЕКТИВНОСТИ ЭКСТРАКОРПОРАЛЬНОГО ОПЛОДОТВОРЕНИЯ
}

\author{
А. К. Рабаданова, Р. И. Шалина, Н. А. Гугушвили \\ Кафедра акушерства и гинекологии, педиатрический факультет, \\ Российский национальный исследовательский медицинский университет имени. Н. И. Пирогова
}

\begin{abstract}
Эффрективность программы экстракорпорального оплодотворения (ЭКО) зависит от ряда фракторов, среди которых большое значение имеют эмбриональный и эндометриальный. Каждый из этих факторов напрямую зависит от овариального резерва, определяющего качество ооцитов и изменение допплерометрических показателей в матке, которые отражают снижение внутриматочной перфузии. Целью исследования было прогнозирование эффективности циклов ЭКО и переноса эмбрионов (ПЭ) у пациенток с различным овариальным резервом на основании определения объемногокровотокавматкевденьпереносаэмбрионаспомощью3D-УЗИсфункциейэнергетическойдопплерограсии. У 56 пациенток с различным овариальным резервом произведено измерение параметров кровотока с помощью 3D-УЗИ и прикладной программы VOCAL (Virtual Organ Computer-aided AnaLysis) в матке, субэндометриальной зоне и в эндометрии в день переноса эмбриона с последующей оценкой эффективности циклов ЭКО. В ходе работы установлена зависимость между параметрами 3D-кровотока в матке и субэндометриальной зоне и эффективностью циклов ЭКО и ПЭ. При исследовании кровотока в эндометрии такая зависимость не выявлена. Таким образом, использование 3D-УЗИ с функцией энергетической допплерографии в день переноса эмбриона позволяет судить о степени выраженности рецептивности эндометрия и может быть использовао в качестве прогностического критерия для определения вероятности наступления беременности в цикле ЭКО.
\end{abstract}

Ключевые слова: экстракорпоральное оплодотворение, сниженный овариальный резерв, маточный кровоток, 3D-УЗИ с функцией энергетической допплерографии

Для корреспонденции: Асият Рабаданова

ул. Мнёвники, д. 23, к. 254, г. Москва, 123423; asyakubataeva@gmail.com

Статья получена: 15.03.2018 Статья принята к печати: 20.03.2018

DOI: $10.24075 /$ vrgmu.2018.015

\section{UTERINE HEMODYNAMICS AND OVARIAN RESERVE QUALITY IN THE PREDICTION OF IN VITRO FERTILIZATION OUTCOMES}

\author{
Rabadanova AK, $\bowtie$ Shalina RI, Gugushvili NA
}

Department of Obstetrics and Gynecology, Faculty of Pediatrics,

Pirogov Russian National Research Medical University, Moscow

\begin{abstract}
There are a few major factors determining the success of in vitro fertilization (IVF), including the potential of embryos to implant and the receptivity of the endometrium, which, in turn, are directly dependent on the ovarian reserve, i.e. the quality and quantity of oocytes. Diminished ovarian reserve can be inferred from reduced blood flow to the uterus on Doppler sonography. Based on the results of 3D power Doppler imaging of uterine blood flow on the day of ET and Virtual Organ Computer-aided AnaLysis (VOCAL), we attempted to predict the outcomes of IVF and embryo transfer (ET) in 56 female patients with different ovarian reserves. Blood flow was measured in the uterus, subendometrial region and endometrium, and IVF cycle outcomes were subsequently assessed. We have established an association between the characteristics of uterine and subendometrial blood flow and the outcomes of IVF cycles and ET. No such association has been established for the endometrium, though. Therefore, a 3D power Doppler examination on the day of embryo transfer provides valuable information on the endometrium receptivity and can be used as a prognostic marker of IVF success.
\end{abstract}

Keywords: IVF, 3D power Doppler, diminished ovarian reserve, poor ovarian response, uterine blood flow

Correspondence should be addressed: Asiyat Rabadanova

Mnevniki 23, k. 254, Moscow, 123423; asyakubataeva@gmail.com

Received: 15.03.2018 Accepted: 20.03.2018

DOI: 10.24075/brsmu.2018.015

Согласно современным представлениям, эффективность программы ЭКО в основном зависит от двух фракторов: способности эмбриона к внутриматочной нидации (эмбриональный фактор) и качества эндометрия (эндометриальный фактор) [1]. Каждый из этих факторов напрямую зависит от овариального резерва. Так, при сниженном овариальном резерве отмечается тенденция к ухудшению качества ооцитов [2] и изменению допплерометрических показателей, отражающих снижение внутриматочной перфузии. В связи с тем, что в 
исследовании изучали кровоснабжение матки, а в циклах ЭКО переносили только эмбрионы хорошего качества на 5е сутки, внимание было обращено именно на эндометриальный фактор.

В отчете Российской ассоциации репродукции человека (РАРЧ) за 2013 г. показано, что замена собственных эмбрионов на донорские у пациенток после 40 лет в протоколе криоконсервации увеличивает эффективность циклов ЭКО всего на 4,8\% [3]. Представленные данные косвенно указывают на то, что повышение эффективности программ ЭКО у пациенток со сниженным овариальным резервом в значительной степени зависит от эндометриального фактора. В связи с этим оценка состояния рецептивности эндометрия и поиск подходов к ее повышению являются актуальной сферой исследования. 3D-УЗИ с функцией энергетической допплерографии может быть отличной неинвазивной методикой, по результатам которой можно косвенно судить о рецептивности эндометрия $[4,5]$.

Пролиферация и дифференциация эндометрия контролируются стероидными гормонами яичника, отвечающими за активный ангиогенез [6], который играет важнейшую роль в росте эндометрия и последующей имплантации эмбриона $[7,8]$. Адекватное кровоснабжение эндометрия и субэндометриальный зоны рассматривается как необходимое условие для успешной имплантации.

В ходе исследования, проведенного в 2012 г. [8], авторы сравнивали кровоснабжение субэндометриальной зоны с помощью 2D- и 3D-УЗИ и полученные результаты соотносили с данными о рецептивности эндометрия, определяющей «окно имплантации», полученными с помощью пайпель-биопсии. Было выявлено, что 3D-УЗИ по сравнению c 2D-УЗИ обладает большей прогностической способностью и является более точным методом, с помощью которого возможно прогнозирование состояния эндометрия. На отсутствие зависимости между результатами 2D-УЗИ и эффективностью циклов ЭКО указывали и другие исследователи [9].

Работ, посвященных оценке объемного кровотока матки у пациенток с различным овариальным резервом, определяемого для прогнозирования частоты наступления беременности в циклах ЭКО и ПЭ, недостаточно.

Целью исследования было прогнозирование вероятности наступления беременности у пациенток с различным овариальным резервом в цикле ЭКО на основании определения объемного кровотока в матке в день переноса эмбриона с помощью 3D-УЗИ.

\section{ПАЦИЕНТЫ И МЕТОДЫ}

Исследование проводили на базе отделения репродукции ЭКО Центра планирования семьи и репродукции (ЦПСиР) с 2015 по 2017 г.

В рамках исследования проводили проспективный анализ лечебных стимулируемых циклов стандартных программ ЭКО у 56 пациенток с различным овариальным резервом, в ходе которых выполняли перенос на 5-е сутки не более двух эмбрионов на стадии бластоцисты. В зависимости от эффективности проведенного цикла выделили две группы. Первую составили пациентки после ЭКО с наступившей беременностью (21 пациентка), вторую - те, у кого после лечебного цикла беременность не наступила (35 пациенток). Среди пациенток с наступившей беременностью у 13 (61,9\%) был трубноперитонеальный фактор бесплодия, у 8 (38,1\%) - сниженный овариальный резерв. У 23 (65,7\%) пациенток с неэффективным циклом ЭКО был трубно-перитонеальный фактор бесплодия, у 12 (34,2\%) - сниженный овариальный резерв.

Критерии включения: возраст более 30 лет и менее 40 лет; фактор бесплодия - сниженный овариальный резерв и трубно-перитонеальный фактор.

Критерии исключения: наличие объемных образований яичников, миомы матки, аденомиоз; фактор бесплодия у партнера; проведенный курс терапии, направленной на улучшение маточного кровотока.

О степени выраженности снижения овариального резерва судили по уровню фолликулостимулирующего (ФСГ) и антимюллерова гормона (АМГ), количеству антральных фолликулов до 10 мм в диаметре, выявленных при УЗИ на 2-3-й день лечебного цикла. Если уровень ФСГ превышал 8 MЕ/л, уровень АМГ был ниже 1 нг/мл и количество полученных ооцитов после трансвагинальной пункции достигало 3 и менее, то овариальный резерв считали сниженным.

Для стимуляции яичников в циклах стандартных программ ЭКО использовали протокол с антагонистом гонадотропин-релизинг гормона. Стимуляцию овуляции проводили рекомбинантным фолликулостимулирующим гормоном (рФСГ).

Подбор стартовой дозы ФСГ, входящей в состав препаратов для стимуляции суперовуляции (от 75 до 275 ME/сут.), осуществляли индивидуально в зависимости от возраста, индекса массы тела (ИМТ), уровня ФСГ и АМГ, а также на основании ответа яичников на предыдущие циклы стимуляции. Контроль за индуцированным фолликулогенезом проводили с помощью УЗИ. При достижении лидирующим фолликулом диаметра 18-20 мм вводили триггер овуляции хорионический гонадотропин человека (ХГЧ) и через 34-36 ч выполняли трансвагинальную пункцию фолликулов диаметром больше 15 мм. Выделение ооцитов, их инсеминацию, культивирование и ПЭ, а также поддержку лютеиновой фазы препаратами прогестеронового ряда в посттрансферном периоде выполняли в соответствии с существующими стандартными рекомендациями [2] .

В день переноса эмбриона осуществляли 3D-реконструкцию матки с использованием функции энергетической допплерографии. УЗИ проводили на приборе Voluson 730 Expert (GE Healthcare, Austria) c использованием объемного трансвагинального датчика с частотой 5-9 МГц. Так как от различной частоты параметры кровотока у одной и той же пациентки меняются, нами были установлены следующие настройки аппарата: Gn 9,0; Frq - low; Qual — norm; WMF — low1; PRF — 0,9 кГц.

Для 3D-сканирования получали 2D-изображение с заданным на нем окном объемной реконструкции. Первичное 2D-изображение соответствовало центральной плоскости сканируемого объема [10, 11], а само сканирование разворачивали от одной его границы до другой. Угол захвата объема составлял $120^{\circ}$ с последующим шагом обведения $15^{\circ}$ и не более, поскольку при малой дискретности сосуды, расположенные рядом с маткой, могут попадать в объем реконструкции объекта.

При помощи прикладной программы VOCAL в ручном режиме производили обводку контура матки и эндометрия. Оценку особенностей васкуляризации эндометрия проводили двумя методами. Первый включал в себя обводку эндометрия по границе базального слоя. При втором методе граница контура обводки находилась на 5 мм 
кнаружи от базального слоя, в области субэндометрия включая его, для захвата не только базальних, но и радиальных артерий [12]. Погрешностью при построении считали зазубренный контур в некоторых срезах органа и в целом, несмотря на корректную обводку каждого среза. После проведенных манипуляций автоматически рассчитывали объем матки, эндометрия и субэндометрия. При построении гистограмм определяли такие показатели, как индекс васкуляризации (vascularization index, VI), характеризующий процентное соотношение сосудов в определенном объеме ткани; индекс потока (flow index, FI), который показывает объем крови, перемещающейся в сосудах во время исследования; васкуляризационнопотоковый индекс (vascularization-flow index, VFI), являющийся показателем перфузии органа.

Полученные результаты обрабатывали с использованием пакетов прикладных программ Excel 7.0, Statistica 6.0. Различия частот анализируемого признака при парных сравнениях считали достоверным при уровне значимости p < 0,05. Для обработки данных использовали также $\mathrm{ROC}$-анализ с определением площади под кривой. Чем выше значение площади под ROC-кривой, тем лучше качество модели: 0,9-1,0 - отличное; 0,8-0,9 - очень хорошее; 0,7-0,8 - хорошее; 0,6-0,7 - среднее; 0,5-0,6 неудовлетворительное.

\section{РЕЗУЛЬТАТЫ ИССЛЕДОВАНИЯ}

Возраст пациенток варьировал от 30 до 42 лет (34,27 \pm 3,98 года). Среди них до 35 лет было 17 пациенток, от 35 до 42 лет - 39 пациенток. Возраст пациенток, у которых после ПЭ наступила беременность (I группа), составил в среднем 34,27 $\pm 3,98$ года. При ненаступившей беременности (II группа) - 36,00 \pm 3,74 года $(p>0,05)$. Несмотря на тенденцию повышения возраста у пациенток с неэффективным циклом по сравнению с эффективным, особенно у пациенток со сниженным овариальным резервом, достоверных различий этого параметра не отмечено (табл. 1).

Благодаря тщательному отбору пациенток, проходящих лечение методом вспомогательной репродуктивной технологии (ВРТ), количество случаев возникновения экстрагенитальной патологии не выходило за рамки общепопуляционных.

При оценке характера гинекологической патологии отмечалось относительно большое количество случаев оперативных вмешательств (см. табл. 1) на яичниках (40\%) у пациенток со сниженным овариальным резервом и отсутствием эффекта от ЭКО. Показаниями к кистэктомии было наличие цистаденомы различных размеров у 5 пациенток (31,2\%), эндометриоидных кист - у 11 (68,8\%).

При неудачной попытке ЭКО как при сниженном, так и при нормальном овариальном резерве у половины пациенток в анамнезе наблюдалось раздельное диагностическое выскабливание слизистой матки по поводу гиперпластических процессов в эндометрии (17-48,6\%); из них у 14 пациенток - по поводу полипа эндометрия и у 3 пациенток - по поводу гиперплазии эндометрия. При эффективном цикле случаи патологии эндометрия встречались в 1,7 раза реже (см. табл. 1).

Стаж бесплодия варьировал в широких границах от 1 года до 11 лет. У пациенток со сниженным овариальным резервом, как в I группе, так и во II, он был относительно выше, чем у пациенток с нормальным овариальным резервом, но достоверно не отличался (см. табл. 1).

У пациенток с наступившей беременностью первичное бесплодие было отмечено у большинства. При неэффективном цикле ЭКО первичное бесплодие тоже было у большинства пациенток (у 20-57,1\%).

Уровни ФСГ и АМГ, определяющие функцию яичников, достоверно различались в зависимости от овариального резерва. Так, низкий уровень АМГ и высокий уровень

Таблица 1. Характеристика пациенток при различном овариальном резерве и эффективность ЭКО

\begin{tabular}{|c|c|c|c|c|c|}
\hline & \multicolumn{2}{|c|}{$\begin{array}{c}\text { Эффективный цикл } \\
(\mathrm{n}=21)\end{array}$} & \multicolumn{2}{|c|}{$\begin{array}{l}\text { Неэффективный цикл } \\
(\mathrm{n}=35)\end{array}$} & \multirow[b]{2}{*}{$p$} \\
\hline & $\begin{array}{c}\text { Хороший } \\
\text { овариальный резерв } \\
(n=13) p^{1}\end{array}$ & $\begin{array}{c}\text { Сниженный } \\
\text { овариальный резерв } \\
(\mathrm{n}=8) \mathrm{p}^{2}\end{array}$ & $\begin{array}{c}\text { Хороший } \\
\text { овариальный резерв } \\
(n=23) p^{3}\end{array}$ & $\begin{array}{c}\text { Сниженный } \\
\text { овариальный резерв } \\
(n=12) p^{4}\end{array}$ & \\
\hline Возраст (лет) & $33,27 \pm 4,98$ & $35,45 \pm 2,98$ & $35,09 \pm 3,46$ & $37,02 \pm 2,87$ & - \\
\hline Индекс массы тела & $24,36 \pm 1,98$ & $24,76 \pm 1,43$ & $25,02 \pm 1,17$ & $25,32 \pm 1,87$ & - \\
\hline Стаж бесплодия (лет) & $4,16 \pm 2,54$ & $5,26 \pm 3,54$ & $5,98 \pm 2,07$ & $5,41 \pm 3,07$ & - \\
\hline Первичное бесплодие & $\begin{array}{c}3 \\
14,3 \%\end{array}$ & $\begin{array}{c}6 \\
28,6 \%\end{array}$ & $\begin{array}{c}5 \\
14,3 \%\end{array}$ & $\begin{array}{c}10 \\
28,6 \%\end{array}$ & - \\
\hline Вторичное бесплодие & $\begin{array}{c}10 \\
47,6 \%\end{array}$ & $\begin{array}{c}2 \\
9,5 \%\end{array}$ & $\begin{array}{c}18 \\
51,4 \%\end{array}$ & $\begin{array}{c}2 \\
5,7 \%\end{array}$ & - \\
\hline $\begin{array}{l}\text { Уровень ФСГ } \\
\text { (мМЕ/мл) }\end{array}$ & $6,8 \pm 1,56$ & $11,99 \pm 7,03$ & $7,18 \pm 1,79$ & $12,29 \pm 5,99$ & $\begin{array}{l}p^{1 / p^{2}}=0,001^{*} \\
p^{3} / p^{4}=0,002^{*}\end{array}$ \\
\hline $\begin{array}{l}\text { Уровень АМГ } \\
\text { (нг/мл) }\end{array}$ & $3,26 \pm 1,27$ & $0,61 \pm 0,42$ & $3,57 \pm 2,43$ & $0,65 \pm 0,37$ & $\begin{array}{l}\mathrm{p}^{1 / \mathrm{p}^{3}}=0,021^{*} \\
\mathrm{p}^{2} / \mathrm{p}^{4}=0,001^{*}\end{array}$ \\
\hline Доза рФСГ (МЕ) & $2202,5 \pm 986,8$ & $1602,9 \pm 796,8$ & $2309,2 \pm 862,2$ & $1809,2 \pm 987,8$ & - \\
\hline $\begin{array}{l}\text { Толщина эндометрия в день } \\
\text { переноса (мм) }\end{array}$ & $10,4 \pm 2,6$ & $10,60 \pm 1,94$ & $8,4 \pm 0,9$ & $8,31 \pm 1,28$ & $\begin{array}{l}\mathrm{p}^{1} / \mathrm{p}^{3}=0,05^{*} \\
\mathrm{p}^{2} / \mathrm{p}^{4}=0,003^{*}\end{array}$ \\
\hline $\begin{array}{l}\text { Количество перенесенных } \\
\text { эмбрионов }\end{array}$ & $1,49 \pm 0,21$ & $1,59 \pm 0,29$ & $1,76 \pm 0,68$ & $1,32 \pm 0,43$ & - \\
\hline Операции на яичниках & $\begin{array}{c}1 \\
4,8 \%\end{array}$ & $\begin{array}{c}3 \\
14,9 \%\end{array}$ & $\begin{array}{c}5 \\
14,9 \%\end{array}$ & $\begin{array}{c}7 \\
40 \%\end{array}$ & - \\
\hline $\begin{array}{l}\text { Количество случаев патологии } \\
\text { эндометрия в анамнезе }\end{array}$ & $\begin{array}{c}4 \\
19,0 \%\end{array}$ & $\begin{array}{c}2 \\
9,5 \%\end{array}$ & $\begin{array}{c}9 \\
25,7 \%\end{array}$ & $\begin{array}{c}8 \\
22,9 \%\end{array}$ & - \\
\hline
\end{tabular}

Примечание: * — показатели с достоверными различиями 
ФСГ отмечался у пациенток со сниженным овариальным резервом (см. табл.1).

Дозы рФСГ и количество перенесенных эмбрионов у пациенток в обеих группах достоверно не различались.

На 5-й день после трансвагинальной пункции перед переносом эмбриона были осуществлены 2D- и 3D-УЗИ с функцией энергетической допплерографии

В нашем исследовании при оценке толщины эндометрия в день переноса эмбриона у пациенток с наступившей беременностью данный параметр варьировал от 8,0 до 14,5 мм. Самый большой показатель толщины эндометрия (14,5 мм) отмечен после переноса эмбриона пациентке, у которой впоследствие родилась двойня. При неэффективных циклах ЭКО толщина эндометрия варьировала от 7,0 до 10,6 мм. При сравнении зависимости данного параметра от функций яичника отмечено, что достоверные различия значений между группами с эффективным и неэффективным циклом ЭКО были у пациенток как со сниженным, так и с хорошим овариальным резервом ( $p=0,003 ; p=0,05)$ (см. табл. 1).

Следовательно, значение толщины эндометрия в день переноса эмбриона может служить достаточно информативным критерием эффективности цикла ЭКО как при хорошем овариальном резерве, так и при сниженном

При исследовании объема матки с помощью 3D-УЗИ в группе с наступившей беременностью (I группа) размеры ее варьировали от 31,7 до 83,3 мм³ , в группе с неэффективным циклом - от 21,0 до 90,3 мм³. По результатам анализа, обнаружена тенденция к изменению объема матки в зависимости от наличия родов в анамнезе. Так, при объеме матки более 58 мм³ у 87,5\% пациенток в | группе и у 65,7\% во || группе в анамнезе имелись роды. Не отмечена зависимость объема матки от овариального резерва и эффективности цикла ЭКО (p > 0,05) (табл. 2).

Среднее значение объема субэндометриальной зоны при эффективном цикле ЭКО варьировало от 3,5 до 13,9 мм³ . Показатели объема у женщин с ненаступившей

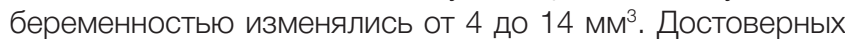
различий средних значений этого показателя в зависимости от эффективности циклов ЭКО и овариального резерва не отмечено (р > 0,05) (табл. 2).

Таким образом, проведенное исследование показало, что значения объемов миометрия и субэндометриальной зоны значимости в прогнозировании эффективности циклов ЭКО как при сниженном, так и при нормальном овариальном резерве не имеют.

В современной литературе имеются данные о необходимости изучения объема эндометрия, учитывая большую корреляцию его показателей с исходами циклов ЭКО. Рекомендуется учитывать значения именно объема эндометрия в цикле ЭКО, а не его толщину (М-эхо), в связи с большой субъективностью второго показателя [12]. В нашем исследовании выявлено, что как при сниженном, так и при нормальном овариальном резерве, объем эндометрия у пациенток с эффективным циклом ЭКО был достоверно больше, чем у пациенток с ненаступившей беременностью ( эффективном цикле ЭКО и нормальном овариальном резерве данный параметр был в 1,4 и 1,7 раза выше, чем при неэффективном ( $p=0,004, p=0,008)$ (см. табл. 2). В связи с полученными данными объем эндометрия можно использовать в качестве критерия эффективности цикла ЭКО.

При индивидуальном анализе выявлено, что у пациенток, имеющих в анамнезе раздельное диагностическое выскабливание слизистой матки, в день переноса эмбриона отмечались относительно низкие значения объема эндометрия как при эффективном, так и при неэффективном цикле, в отличии от пациенток без оперативного вмешательства.

Для прогнозирования эффективности циклов ЭКО применяли метод энергетической допплерографии с оценкой низкоскоростных потоков крови, которые характерны для миометрия и эндометрия. По некоторым данным, именно эта методика увеличивает количество визуализируемых сосудов с низкой скоростью кровотока [11].

При оценке индексов кровотока, полученных в ходе построения гистограмм (табл. 3), выявлена следующая закономерность: индексы объемного кровотока в миометрии и субэндометрии были достоверно выше у пациенток с наступившей беременностью после ПЭ. Данные, представленные в табл. 3, свидетельствуют о том, что индекс васкуляризации (VI) и васкуляризационнопотоковый индекс (VFI) в миометрии при эффективном цикле ЭКО были в 1,6 и 2,0 раза выше, чем при отсутствии эффекта от ЭКО. Аналогичные изменения отмечены при анализе васкуляризации субэндометриальной зоны. Наибольшие различия в значениях имеют эти же параметры VI и VFI: они в 2,6 и 2,5 раза выше у пациенток с наступившей беременностью после ПЭ.

При сравнении изменений параметров кровотока у пациенток в зависимости от овариального резерва установлено, что при неэффективном цикле ЭКО и сниженном овариальном резерве VI и VFI в миометрии были в 1,6 и 1,5 раза, а VFI в субэндометриальной зоне в 2,5 раза ниже, чем при нормальном овариальном резерве и неэффективном цикле ( $<<0,05)$ (табл. 3). Указанное свидетельствует о пониженном кровенаполнении матки у пациентов со сниженным овариальным резервом в отличие от женщин с адекватной функцией яичников.

Таблица 2. Показатели объема миометрия, субэндометриальной зоны и эндометрия, полученные при 3D-УзИ

\begin{tabular}{|c|c|c|c|c|c|}
\hline & \multicolumn{2}{|c|}{$\begin{array}{c}\text { Эффективный цикл } \\
(\mathrm{n}=21)\end{array}$} & \multicolumn{2}{|c|}{$\begin{array}{l}\text { Неэффективный цикл } \\
(\mathrm{n}=35)\end{array}$} & \multirow[t]{2}{*}{$\mathrm{p}$} \\
\hline & $\begin{array}{c}\text { Хороший овариальный } \\
\text { резерв }\left(\mathrm{MM}^{3}\right) \\
(\mathrm{n}=13) \\
\mathrm{p}^{1}\end{array}$ & $\begin{array}{c}\text { Сниженный овариальный } \\
\text { резерв }\left(\text { мм }^{3}\right) \\
(\mathrm{n}=8) \\
\mathrm{p}^{2}\end{array}$ & $\begin{array}{c}\text { Хороший овариальный } \\
\text { резерв }\left(\text { мм }^{3}\right) \\
(\mathrm{n}=25) \\
\mathrm{p}^{3}\end{array}$ & $\begin{array}{c}\text { Сниженный овариальный } \\
\text { резерв }\left(\text { мм }^{3}\right) \\
(\mathrm{n}=12) \\
\mathrm{p}^{4}\end{array}$ & \\
\hline Объем миометрия & $49,4 \pm 5,01$ & $47,2 \pm 4,98$ & $49,2 \pm 3,67$ & $48,6 \pm 5,12$ & - \\
\hline Объем субэндометрия & $10,0 \pm 1,1$ & $9,8 \pm 0,8$ & $8,9 \pm 0,8$ & $8,7 \pm 0,7$ & - \\
\hline Объем эндометрия & $2,53 \pm 0,19$ & $1,38 \pm 0,31$ & $1,79 \pm 0,38$ & $0,82 \pm 0,25$ & $\begin{array}{l}\mathrm{p}^{1} / \mathrm{p}^{2}=0,004^{*} \\
\mathrm{p}^{3} / \mathrm{p}^{4}=0,008^{*} \\
\mathrm{p}^{1} / \mathrm{p}^{3}=0,05^{*} \\
\mathrm{p}^{2} / \mathrm{p}^{4}=0,02^{*}\end{array}$ \\
\hline
\end{tabular}

Примечание: * 一 показатели с достоверными различиями. 
Таблица 3. Показатели гемодинамики в матке при различном овариальном резерве и эффективность ЭКО

\begin{tabular}{|c|c|c|c|c|c|c|c|c|c|c|}
\hline \multirow{2}{*}{ Группы } & \multirow{2}{*}{$\begin{array}{c}\text { Эффективность/ } \\
\text { Параметры } \\
\text { ЗD-УЗИ }\end{array}$} & \multicolumn{3}{|c|}{ В матке } & \multicolumn{3}{|c|}{ В субэндометрии } & \multicolumn{3}{|c|}{ В эндометрии } \\
\hline & & VI & $\mathrm{FI}$ & VFI & $\mathrm{VI}$ & $\mathrm{FI}$ & VFI & $\mathrm{VI}$ & $\mathrm{FI}$ & VFI \\
\hline \multirow{2}{*}{  } & $\begin{array}{c}\text { Эффективный } \\
\text { цикл } \\
(\mathrm{n}=13) \\
\mathrm{p}^{1} \\
\end{array}$ & $11,41 \pm 2,19$ & $16,16 \pm 2,90$ & $1,77 \pm 0,35$ & $6,13 \pm 4,23$ & $10,0 \pm 1,17$ & $0,92 \pm 0,64$ & $3,41 \pm 2,82$ & $11,60 \pm 1,61$ & $0,32 \pm 0,33$ \\
\hline & $\begin{array}{c}\text { Неэффективный } \\
\text { цикл } \\
(\mathrm{n}=25) \\
\mathrm{p}^{2}\end{array}$ & $7,45 \pm 0,97$ & $12,75 \pm 1,68$ & $0,94 \pm 0,05$ & $3,19 \pm 2,57$ & $10,55 \pm 1,90$ & $0,25 \pm 0,15$ & $2,5 \pm 2,22$ & $11,38 \pm 2,76$ & $0,27 \pm 0,21$ \\
\hline \multirow{2}{*}{ 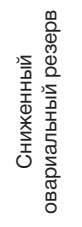 } & $\begin{array}{c}\text { Эффективный } \\
\text { цикл } \\
(\mathrm{n}=8) \\
\mathrm{p}^{3} \\
\end{array}$ & $10,56 \pm 2,57$ & $17,68 \pm 3,44$ & $1,92 \pm 0,75$ & $3,52 \pm 1,09$ & $11,12 \pm 1,03$ & $0,39 \pm 0,16$ & $2,40 \pm 1,84$ & $10,69 \pm 1,68$ & $0,22 \pm 0,20$ \\
\hline & $\begin{array}{c}\text { Неэффективный } \\
\text { цикл } \\
(\mathrm{n}=12) \\
\mathrm{p}^{4}\end{array}$ & $4,47 \pm 2,30$ & $14,05 \pm 3,32$ & $0,62 \pm 0,28$ & $1,23 \pm 1,27$ & $8,61 \pm 3,95$ & $0,10 \pm 0,08$ & $1,82 \pm 1,49$ & $11,17 \pm 2,16$ & $0,19 \pm 0,16$ \\
\hline \multicolumn{2}{|r|}{$p$} & $\begin{array}{l}\mathrm{p}^{1} / \mathrm{p}^{2}=0,007^{\star \star} \\
\mathrm{p}^{3} / \mathrm{p}^{4}=0,003^{\star \star} \\
\mathrm{p}^{1 / \mathrm{p}^{3}}=0,62 \\
\mathrm{p}^{2} / \mathrm{p}^{4}=0,014^{\star}\end{array}$ & $\begin{array}{l}p^{1 / p^{2}}=0,06 \\
p^{3} / p^{4}=0,35 \\
p^{1 / p^{3}}=0,52 \\
p^{2} / p^{4}=0,41\end{array}$ & $\begin{array}{l}\mathrm{p}^{1 / p^{2}}=0,23 \\
\mathrm{p}^{3} / \mathrm{p}^{4}=0,009^{\star \star} \\
\mathrm{p}^{1 / \mathrm{p}^{3}}=0,78 \\
\mathrm{p}^{2} / \mathrm{p}^{4}=0,011^{\star}\end{array}$ & $\begin{array}{l}\mathrm{p}^{1 / 1} \mathrm{p}^{2}=0,24 \\
\mathrm{p}^{3} / \mathrm{p}^{4}=0,014^{*} \\
\mathrm{p}^{1 / \mathrm{p}^{3}}=0,27 \\
\mathrm{p}^{2} / \mathrm{p}^{4}=0,12\end{array}$ & $\begin{array}{l}\mathrm{p}^{1 / \mathrm{p}^{2}}=0,58 \\
\mathrm{p}^{3 / \mathrm{p}^{4}}=0,18 \\
\mathrm{p}^{1 / \mathrm{p}^{3}}=0,19 \\
\mathrm{p}^{2} / \mathrm{p}^{4}=0,30\end{array}$ & $\begin{array}{l}\mathrm{p}^{1} / \mathrm{p}^{2}=0,013^{*} \\
\mathrm{p}^{3} / \mathrm{p}^{4}=0,007^{* *} \\
\mathrm{p}^{1 / \mathrm{p}^{3}}=0,12 \\
\mathrm{p}^{2} / \mathrm{p}^{4}=0,04^{*}\end{array}$ & $\begin{array}{l}p^{1 / p^{2}}=0,34 \\
p^{3} / p^{4}=0,82 \\
p^{1 / p^{3}}=0,21 \\
p^{2} / p^{4}=0,41\end{array}$ & $\begin{array}{l}p^{1} / p^{2}=0,83 \\
p^{3} / p^{4}=0,65 \\
p^{1 / p^{3}}=0,37 \\
p^{2} / p^{4}=0,85\end{array}$ & $\begin{array}{l}\mathrm{p}^{1 / p^{2}}=0,57 \\
\mathrm{p}^{3 / p^{4}}=0,77 \\
\mathrm{p}^{1 / p^{3}}=0,57 \\
\mathrm{p}^{2} / \mathrm{p}^{4}=0,32\end{array}$ \\
\hline
\end{tabular}

Примечания: * — показатели с достоверными различиями при $\mathrm{p}<0,05$; ${ }^{*}$ — показатели с достоверными различиями при $\mathrm{p}<0,01$.

Напротив, при эффективном цикле ЭКО, сравнение пациенток с различным овариальным резервом не выявило различий в параметрах кровотока как при сниженном, так и при нормальном овариальном резерве.

Для сравнения двух методов оценки кровоснабжения эндометрия (рис. 1) (с захватом субэндометриальной зоны и без) проводили ROC-анализ (рис. 2), в ходе которого было выявлено, что при обведении эндометрия строго по базальному контуру (кровоток в эндометрии) зависимости показателей кровотока и эффективности циклов ЭКО не было получено (рис. 2 а) (площадь под кривой для каждого измеренного параметра: для VI - 0,49; для $\mathrm{FI}-0,50$; для $\mathrm{VFI}-0,49)$.

При обведении контура на 5 мм кнаружи от базального слоя с захватом субэндометриальной зоны отмечена зависимость между параметрами кровотока и эффективностью циклов ЭКО (рис. 2 б) (площадь под кривой для каждого измеренного параметра: для $\mathrm{VI}-$ 0,88; для $\mathrm{FI}-0,66$; для VFI - 0,91).

При анализе полученных нами данных с помощью 2D- и 3D-УЗИ выделены основные параметры, имеющие достоверную корреляцию с эффективностью цикла ЭКО независимо от овариального резерва: объем эндометрия, индекс VI миометрия и VFI субэндометрия. Прогностическая значимость данных показателей представлена с помощью ROC-кривой (рис. 3). Чувствительность 74,8\%, специфичность 60,6\%, площадь под кривой 0,851 для объема эндометрия; те же показатели равные 86,4\%, 69,4\%, 0,857 соответственно - для VI миометрия, те же показатели равные 90,4\%, 79,4\%, 0,916 соответственно для VFI субэндометриальной зоны.

\section{ОБСУЖДЕНИЕ РЕЗУЛЬТАТОВ}

При 2D-УЗИ определяли толщину эндометрия (М-эхо). Данные о минимально допустимой толщине эндометрия, необходимой для успешного наступления беременности, в литературе неоднозначны. Большинство исследователей отмечали, что при значении толщины эндометрия менее 7 мм беременность после переноса эмбриона не наступала $[7,13,14]$. Однако в литературе имеются указания о наступлении беременности при значении 6 мм и даже 4 мм [15]. При этом толщина эндометрия свыше 14 мм ассоциирована с высоким риском потери беременности [7].

В ходе проведенного нами исследования выявлено, что при 2D-УЗИ показатели толщины эндометрия у пациенток с различным овариальным резервом зависили от эффективности циклов ЭКО. Однако при нормальном овариальном резерве различия имели большую достоверность ( $p=0,003)$ чем при сниженном овариальном резерве ( $p=0,05)$. В литературе имеются данные об отсутствии зависимости между эффективностью циклов

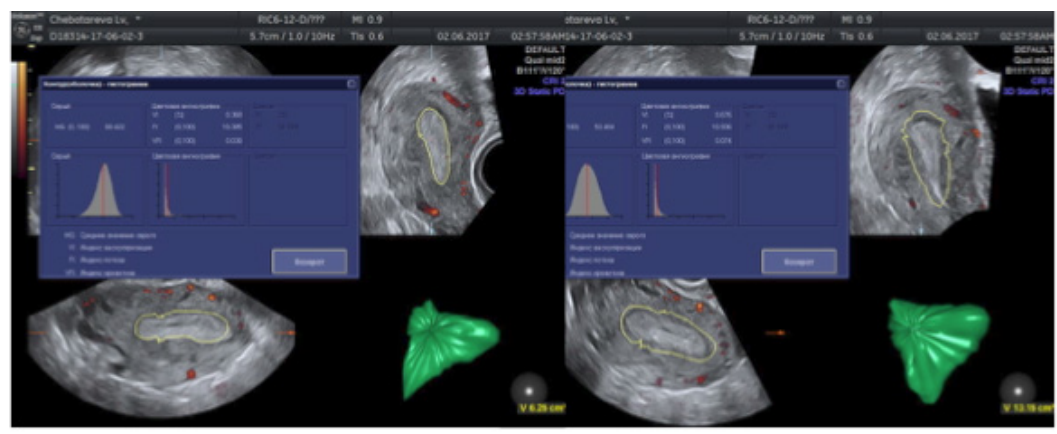

A Кровоснабжение в эндометрии

Б Кровоснабжение в эндометрии с захватом субэндометриальной зоны

Рис. 1. Варианты измерения кровоснабжения в эндометрии и субэндометриальной зоне 




Диагональные сегменты, сгенерированные связями.

A Кровоснабжение в эндометрии

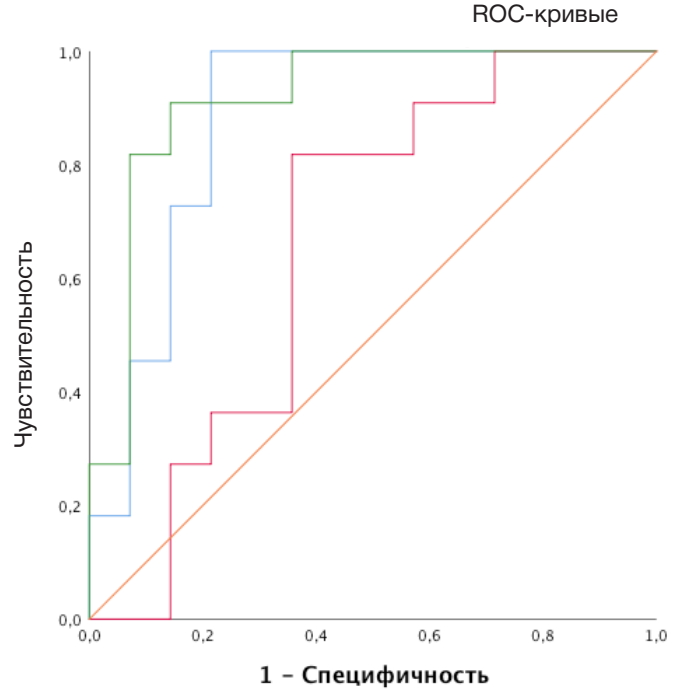

\section{Источник кривой}

- VI субэндометриальной зонь

— Fl субэндометриальной зоны

— VFI субэндометриальной зоны

— Опорная линия

Б Кровоснабжение субэндометриальной зоны

Pиc. 2. ROC-анализ зависимости эффективности циклов ЭКО и кровоснабжения эндометрия (А) и субэндометриальной зоны (Б)

ЭКО и толщиной эндометрия [14]. Сообщалось также, что у пациенток старше 40 лет отмечается тенденция к более низким показателям толщины эндометрия, которая связана со снижением уровня эстрадиола [12]. Ряд авторов утверждают, что толщина эндометрия носит субъективный характер, в связи с чем использование 3D-УЗИ c определением объема эндометрия более показательно и позволяет обнаружить зависимость между этим параметром и эффективностью циклов ЭКО [8]. В нашем исследовании при ЗD-УЗИ показатели объема эндометрия также имели достоверные различия в зависимости от эффективности цикла ( $p=0,05 ; \mathrm{p}=$ 0,02). При сравнении значений объема эндометрия у забеременевших пациенток с различным овариальным резервом отмечена достоверная разница показателей $(p=0,004)$, в то время как при измерении толщины эндометрия данных различий не обнаружено.

Немаловажное значение следует придавать особенностям внутриматочного кровотока. В результате большого метанализа показано, что по данным кровоснабжения в субэндометриальной зоне и эндометрии можно косвенно судить о рецептивности эндометрия [5]
Как известно, кровоснабжение эндометрия осуществляется, в основном, за счет расположенных в нем спиральных артерий, а последние являются продолжением базальных артерий, распологающихся в субэндометрии [11]. Определение параметров кровотока в эндометрии с захватом субэндометриальной зоны (субэндометрий) наиболее показательно поскольку отражает интенсивность кровотока дополнительно в дугообразной артерии и отходящих от нее радиальных ветвях.

Учитывая, что в циклах ЭКО в день переноса эмбриона отсутствует возможность для исследования рецептивности ткани с помощью инвазивных методик в связи с травматизацией эндометрия, использование неинвазивных методов оказывается предпочтительным. При исследовании параметров кровотока в матке и субэндометрии выявлены достоверные различия для пациенток независимо от овариального резерва в показателях VI миометрия ( $p=0,007$ для пациенток с нормальным овариальным резервом; $p=0,003$ для пациенток со сниженным овариальным резервом) и в показателях VFI субэндометрия ( $p=0,013$ для пациенток с нормальным овариальным резервом; $p=0,007$ для 

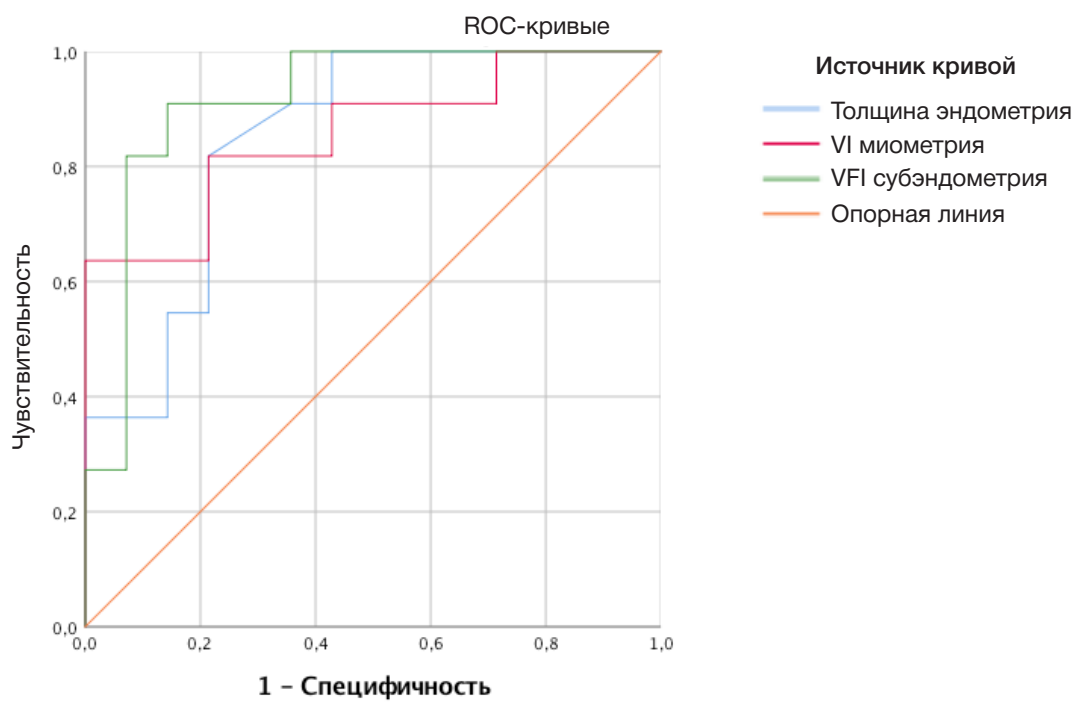

Диагональные сегменты, сгенерированные связями.

Рис. 3. ROC-анализ зависимости эффективности ЭКО и показателей объема эндометрия, VI в миометрии и VFI в субэндометрии

пациенток со сниженным овариальным резервом). Самые низкие параметры кровотоков были отмечены у пациенток с низким овариальным резервом и неэфрфективным циклом ЭКО. Считается, что снижение показателей кровотока при низком овариальном резерве связаны с нарушением роста сосудов функционального слоя эндометрия из-за недостаточного влияния эстрогенов в течение пролисеративной фазы [16].

Подтверждением этих данных могут быть результаты одного из исследований [17], свидетельствующие о том, что содержание эстрогенов в сыворотке крови имеет достоверную связь с интенсивностью маточного кровотока в циклах ЭКО с применением а-ГнРГ и гонадотропинов. Доказано, что при преждевременном истощении функции яичников проведение заместительной гормональной терапии значительно улучшает допплерометрические характеристики кровотока в матке [18].

Таким образом, использование 3D-УЗИ с функцией энергетическойдопплерографиивденьпереносаэмбриона позволяет косвенно судить о степени выраженности рецептивности эндометрия, что согласуется с данными других авторов [5], а также может использоваться в качестве прогностических критериев для определения вероятности наступления беременности после переноса эмбриона в цикле ЭКО.

Информация о показателях кровотока в миометрии и субэндометриальной зоны, а также объем эндометрия позволит избежать заведомо нерезультативных переносов эмбриона. Пациенткам со сниженным овариальным резервом с целью повышения эффективности переноса эмбриона целесообразно исследование кровоснабжения матки и при отклонениях от нормативных показателей рекомендовать проведение терапии, направленной на улучшении маточной перфузии на этапе прегравидарной подготовки.

\section{ВЫВОДЫ}

В нашем исследовании показано, что 3D-УЗИ с функцией энергетической допплерограсии является объективным методом исследования кровоснабжения матки в день переноса эмбриона у пациенток в циклах ЭКО. Для прогнозирования эффективности циклов ЭКО предпочтение следует отдавать параметрам кровотока в миометрии и в субэндометриальном слое. Изучение кровотока в базальном слое эндометрия в нашем исследовании не выявило зависимости с эффективностью цикла ЭКО. Измерение объема эндометрия с помощью 3D-УЗИ в сравнении с определением толщины эндометрия при 2D-УЗИ более информативно и прогностически значимо. Учитывая крайне низкие показатели кровотока при сниженном овариальном резерве и неэффективном цикле ЭКО, ЗД-УЗИ возможно рекомендовать в качестве обязательной процедуры перед переносом эмбриона в цикле ЭКО у данных пациенток.

\section{Литература}

1. Аншина М. Б., Исакова Э. В., Калинина Е. Анат., Калинина Е. Андр., Корсак В. С., Краснопольская К. В. и др. Применение эстрогенов в программах ВРТ. Научно-практические рекомендации. М.: Российская ассоциация репродукции человека. 2015, 80 c.

2. Назаренко Т. А, Краснопольская К. В. «Бедный ответ». Тактика ведения пациенток со сниженной реакцией на стимуляцию гонадотропинами в программах ЭКО. М.: МЕДпресс-информ; 2013, 80 с.

3. Корсак В. С., Смирнова А. А., Шурыгина О. В. ВРТ в России. Отчет за 2013 г. Проблемы репродукции. 2015; 21 (6): 17-19.

4. Mishra W, Agarwal R, Sharma U, Aggarwal R, Choudhary S, Bandwal P. Endometrial and Subendometrial Vascularity by

Three-Dimensional (3D) Power Doppler and Its Correlation with Pregnancy Outcome in Frozen Embryo Transfer (FET) Cycles. $\mathrm{J}$ of Obstetrics and Gynaecology of India. 2016; 66 (Suppl 1): 521-527. DOI: 10.1007/s13224-016-0871-5.

5. Jianing Wang, Fei Xia, Ying Zhou, Xuedong Wei, Yanyan Zhuang, Yingxue Huang. Association Between Endometrial/ Subendometrial Vasculature and Embryo Transfer Outcome: A Meta-analysis and Subgroup Analysis. J Ultrasound Med. 2018 Jan; 37 (1): 149-163. DOI: 10.1002/jum.14319. Epub 2017 Jul 17.

6. Lecce G, Meduri G, Ancelin M. Presence of estrogen receptor bin the human endometrium through the cycle: expression inglandular, stromal, and vascular cells // J Clin Endocrinol. 
Metab. 2001; 86: 1379-86.

7. Amir W, Micha B, Ariel H, Liat LG, Jehoshua D, Adrian S. Predicting factors for endometrial thickness during treatment with assisted reproductive technology. Fertil Steril 2007; 87 (4): 799-804.

8. Kalmantis K, Loutradis D, Lymperopoulos E, Beretsos P, Bletsa $\mathrm{R}$, Antsaklis A. Three Dimensional Power Doppler evaluation of human endometrium after administration of oxytocine receptor antagonist (OTRa ) in an IVF program., Arch Gynecol Obstet. 2012 Jan; 285 (1): 265-70. DOI: 10.1007/s00404-011-2019-2. Epub 2011 Aug 7.

9. Prasad S, Goyal R, Kumar Y, et al. The Relationship Between Uterine Artery two-dimensional Color Doppler Measurement and Pregnancy Outcome: A Prospective Observational Study. Journal of Reproduction \& Infertility. 2017; 18 (2): 251-6.

10. Крылова Ю. С, Кветной И. М., Айламазян Э. К. Рецептивность эндометрия: молекулярные механизмы регуляции имплантации. Журнал акушерства и женских болезней. 2013; 62 (2): 63-74. DOI: http://dx.doi.org/10.17816/JOWD62263-74.

11. Озерская И. А. Атлас гинекологической ультразвуковой нормы. М.: Видар-М; 2010. 225 с.

12. Ari Kim, Hyuk Jung, Won June Choi, Sung Nam Hong, Heung Yeol Kim. Detection of endometrial and subendometrial vasculature on the day of embryo transfer and prediction of pregnancy during fresh in vitro fertilization cycles Taiwanese. Journal of Obstetrics \& Gynecology. 2014; 53 (3): 360-5.

\section{References}

1. Anshina MB, Isakova EHV, Kalinina EAnat, Kalinina EAndr, Korsak VS, Krasnopolskaya KV i dr. Priemenie estrogenov v programmach WPT. Nachno-practicheskie rekomendacii. M. Rossiyskaja associaacija reprodukcii sheloveka. 2015; $80 \mathrm{~s}$.

2. Nazarenko TA, Krasnoposkaya KV. «Poor response.» Tactica vedenija pacientok so snijennoy reacciej a stimuljaciju gonadotropinami v programmach EKO. M. : MEDpress-inform, 2013. 80 s.

3. Korsak VS, Smirnova AA, Shurygina OV. VRT v Rossii. Otchet za 2013 g. Problemy reprodukcii. 2015; 21 (6): 17-19.

4. Mishra W, Agarwal R, Sharma U, Aggarwal R, Choudhary S, Bandwal P. Endometrial and Subendometrial Vascularity by Three-Dimensional (3D) Power Doppler and Its Correlation with Pregnancy Outcome in Frozen Embryo Transfer (FET) Cycles. J of Obstetrics and Gynaecology of India. 2016; 66 (Suppl 1): 521 527. DOI: 10.1007/s13224-016-0871-5.

5. Jianing Wang, Fei Xia, Ying Zhou, Xuedong Wei, Yanyan Zhuang, Yingxue Huang. Association Between Endometrial/ Subendometrial Vasculature and Embryo Transfer Outcome: A Meta-analysis and Subgroup Analysis. J Ultrasound Med. 2018 Jan; 37 (1): 149-163. DOI: 10.1002/jum.14319. Epub 2017 Jul 17.

6. Lecce G, Meduri G, Ancelin M. Presence of estrogen receptor bin the human endometrium through the cycle: expression inglandular, stromal, and vascular cells. J Clin Endocrinol Metab. 2001; 86: 1379-86.

7. Amir W, Micha B, Ariel H, Liat LG, Jehoshua D, Adrian S. Predicting factors for endometrial thickness during treatment with assisted reproductive technology. Fertil Steril 2007; 87 (4): 799-804.

8. Kalmantis K, Loutradis D, Lymperopoulos E, Beretsos P, Bletsa R, Antsaklis A. Three Dimensional Power Doppler evaluation of human endometrium after administration of oxytocine receptor antagonist (OTRa ) in an IVF program., Arch Gynecol Obstet. 2012 Jan; 285 (1): 265-70. DOI: 10.1007/s00404-011-2019-2. Epub 2011 Aug 7.

9. Prasad S, Goyal R, Kumar Y, et al. The Relationship Between Uterine Artery two-dimensional Color Doppler Measurement and
13. Краснопольская К. В., Назаренко Т. А., Сесина Н. И., Ершова И. Ю., Александрова В. Р. Результаты программ ЭКО с донорскими ооцитами у пациенток с ультразвуковыми признаками "тонкого" эндометрия. Медицинский алфавит. М.: Альфмед. 2017; 1 (12) (309) : 34-8.

14. Kasius A, Smit JG, Torrance HL et al. Endometrial thickness and pregnancy rates after IVF: a systematic review and meta-analysis. Hum Reprod Update. 2014; 20 (4): 530-41.

15. Weiss NS, van Vliet MN, Limpens J, Hompes PGA et al. Endometrial thickness in women undergoing IUI with ovarian stimulation. How thick is too thin? A systematic review and metaanalysis. Hum Reprod Update. 2017; 32 (5): 1009-18.

16. Jayaprakasan K, Hopkisson JF, Campbell BK, Clewes J, Johnson IR, Raine-Fenning NJ. Quantification of the effect of pituitary down-regulation on $3 \mathrm{D}$ ultrasound predictors of ovarian response. Human Reproduction. 2008; 23 (7): 1538-44.

17. Bassil S. Changes in endometrial thickness, width, length and pattern in predicting pregnancy outcome during ovarian stimulation in in vitro fertilization. Ultrasound Obstet Gynecol. 2001 Sep; 18 (3): 258-63.

18. Mayra PR, Rosalina VL, López G, Iruretagoyena J, Magness R. Regulation of uterine blood flow. I. Functions of estrogen and estrogen receptor $\alpha / \beta$ in the uterine vascular endothelium during pregnancy. Rev Chil Obstet Ginecol. 2014; 79 (2): 129-39.

Pregnancy Outcome: A Prospective Observational Study. Journal of Reproduction \& Infertility. 2017; 18 (2): 251-6.

10. Krylova YuS, Kvetnoj IM, Ajlamazyan EK. Receptivnost' endometrija: moleculjarnye mechanizmy eguljacii implantacii. J akusherstva i jenskich boleznej. 2013; 62 (2): 63-74. DOI: http:// dx.doi.org/10.17816/JOWD62263-74

11. Ozerskaja IA. Atlas ginekologischeskoj ul'trazvukovoj normy. M.: Vidar-M; 2010. 225 s.

12. Ari Kim, Hyuk Jung, Won June Choi, Sung Nam Hong, Heung Yeol $\mathrm{Kim}$. Detection of endometrial and subendometrial vasculature on the day of embryo transfer and prediction of pregnancy during fresh in vitro fertilization cycles Taiwanese. Journal of Obstetrics \& Gynecology. 2014; 53 (3): 360-5.

13. Krasnopol'skaya KV, Nazarenko TA, Sesina NI, Ershova IYu, Aleksandrova VR. Rezul'taty programmy EKO s donorskimi oocitami u pacientok s ul'trazvukovymi prinakami "tonkogo" endometrija. Medicinskij alfavit. M.: Al'famed. 2017; 1 (12) (309) : 34-8.

14. Kasius A, Smit JG, Torrance $\mathrm{HL}$ et al. Endometrial thickness and pregnancy rates after IVF: a systematic review and meta-analysis. Hum Reprod Update. 2014; 20 (4): 530-41.

15. Weiss NS, van Vliet MN, Limpens J, Hompes PGA et al. Endometrial thickness in women undergoing IUI with ovarian stimulation. How thick is too thin? A systematic review and metaanalysis. Hum Reprod Update. 2017; 32 (5): 1009-18.

16. Jayaprakasan K, Hopkisson JF, Campbell BK, Clewes J, Johnson $I R$, Raine-Fenning NJ. Quantification of the effect of pituitary down-regulation on $3 \mathrm{D}$ ultrasound predictors of ovarian response. Human Reproduction. 2008; 23 (7): 1538-44.

17. Bassil S. Changes in endometrial thickness, width, length and pattern in predicting pregnancy outcome during ovarian stimulation in in vitro fertilization. Ultrasound Obstet Gynecol. 2001 Sep; 18 (3): 258-63.

18. Mayra PR, Rosalina VL, López G, Iruretagoyena J, Magness R. Regulation of uterine blood flow. I. Functions of estrogen and estrogen receptor $\alpha / \beta$ in the uterine vascular endothelium during pregnancy. Rev Chil Obstet Ginecol. 2014; 79 (2): 129-39. 